\title{
Regulation of seed germination and seedling growth by an Arabidopsis phytocystatin isoform, AtCYS6
}

\author{
Jung Eun Hwang $\cdot$ Joon Ki Hong $\cdot$ Ji Hyun Je $\cdot$ \\ Kyun Oh Lee · Dool Yi Kim · Sang Yeol Lee • \\ Chae Oh Lim
}

Received: 25 May 2009/Revised: 27 July 2009/Accepted: 3 August 2009/Published online: 19 August 2009

(C) The Author(s) 2009. This article is published with open access at Springerlink.com

\begin{abstract}
Phytocystatins are cysteine proteinase inhibitors in plants that are implicated in the endogenous regulation of protein turnover and defense mechanisms against insects and pathogens. A cDNA encoding a phytocystatin called AtCYS6 (Arabidopsis thaliana phytocystatin6) has been isolated. We show that AtCYS6 is highly expressed in dry seeds and seedlings and that it also accumulates in flowers. The persistence of AtCYS6 protein expression in seedlings was promoted by abscisic acid (ABA), a seed germination and post-germination inhibitory phytohormone. This finding was made in transgenic plants bearing an AtCYS6 promoter- $\beta$-glucuronidase $(G U S)$ reporter construct, where we found that expression from the AtCYS6 promoter persisted after ABA treatment but was reduced under control conditions and by gibberellin ${ }_{4+7}\left(\mathrm{GA}_{4+7}\right)$ treatment during the germination and post-germinative periods. In addition, constitutive over-expression of AtCYS6 retarded germination and seedling growth, whereas these were enhanced in an AtCYS6 knock-out mutant (cys6-2). Additionally, cysteine proteinase activities stored in seeds were inhibited by
\end{abstract}

Communicated by J. R. Liu.

J.E. Hwang and J.K. Hong contributed equally to this work.

J. E. Hwang - J. K. Hong - J. H. Je - K. O. Lee .

S. Y. Lee · C. O. Lim ( $₫)$

Division of Applied Life Science (BK21 Program),

Environmental Biotechnology National Core Research Center

and PMBBRC, Graduate School of Gyeongsang National

University, Jinju 660-701, Korea

e-mail: colim@gnu.ac.kr

Present Address:

J. K. Hong · D. Y. Kim

National Academy of Agricultural Science,

Rural Development Administration, Suwon 441-707, Korea
AtCYS6 in transgenic Arabidopsis. From these data, we propose that AtCYS6 expression is enhanced by the germination inhibitory phytohormone $\mathrm{ABA}$ and that it participates in the control of germination rate and seedling growth by inhibiting the activity of stored cysteine proteinases.

Keywords Cysteine proteinase inhibitor . GUS expression · Knock-out mutant · Promoter · Transgenic plant

\section{Introduction}

Phytocystatin (PhyCys) are potent inhibitors of cysteine proteinases (CPs) of the papain- (family C1A; MEROPS peptidase database, http://merops.sanger.ac.uk) and legumain-like families (family C13) in plants (Martínez and Díaz 2008). Most PhyCys have a molecular mass in the 12-16 kDa range and contain no disulphide bonds (Gaddour et al. 2001). However, several PhyCys with a molecular mass of $\approx 23 \mathrm{kDa}$ have a carboxy (C)-terminal extension that has been shown to be involved in the inhibition of legumains (Martínez et al. 2007). During seed germination and seedling growth, these legumains act as processing enzymes and contribute to the activation of papain-like CPs to degrade seed storage proteins (Okamoto and Minamikawa 1999; Kato et al. 2003; Zakharov et al. 2004) that serve as precursors for the synthesis of new proteins and other nitrogen-containing compounds in seedlings (Zakharov et al. 2004). PhyCys have also been described as regulators of papain-like CPs due to their ability to inhibit endogenous proteolytic activity during seed germination and seedling growth. The reversible tight binding of PhyCys to papain-like CPs is one of the possible mechanisms by which they control the activity of these peptidases 
(Corre-Menguy et al. 2002; Hong et al. 2007). Numerous studies have attempted to better understand how germination and post-germination are controlled by various hydrolases and inhibitors. However, very little is known about the processes by which the embryo emerges from the seed and the seedling completes growth (Koornneef et al. 2002).

In the present study, we describe the molecular characteristics of Arabidopsis thaliana PhyCys6 (AtCYS6), which has a molecular mass of $22.4 \mathrm{kDa}$ and a C-terminal extension. Our results show that AtCYS6 accumulates in seeds and seedlings and is responsive to the phytohormone ABA. Moreover, constitutive over-expression of AtCYS6 slowed germination and inhibited $\mathrm{CP}$ activity in transgenic Arabidopsis. Additionally, an Arabidopsis knock-out mutant (cys6-2) harboring a T-DNA insertion in the AtCYS6 gene initiated germination earlier than wild-type plants. These findings indicate that AtCYS6 plays a role in the control of seed germination and seedling growth through its ability to regulate $\mathrm{CP}$ activity.

\section{Materials and methods}

Plant materials and growth conditions

Arabidopsis thaliana (L.) Heynh ecotype Columbia (Col-0) plants were grown in soil or in vitro on MS medium (Murashige and Skoog 1962) containing 3\% sucrose and $0.25 \%$ phyta-gel ( $\mathrm{pH} 5.8$ ), under $16 \mathrm{~h}$ of $100 \mu \mathrm{Es}^{-1} \mathrm{~m}^{-2}$ light at $22^{\circ} \mathrm{C}$. To induce synchronous germination, seeds were vernalized at $4^{\circ} \mathrm{C}$ for 3 days in the dark, as previously described (Lim et al. 2007).

A T-DNA insertional mutant line containing a single T-DNA insertion in the AtCYS6 gene was identified in the SALK T-DNA collection (SALK_027847). To identify mutants homozygous for the T-DNA insertion, genomic DNA was obtained from kanamycin-resistant seedlings and subjected to PCR genotyping using the following AtCYS6 primer sets: AtCYS6 P1 primer (5'-ATACAGGGCAC CGAACCAAATAAAG-3') and T-DNA left border P2 primer (5'-GTGATGGTTCACGTAGTGGGCCATCG-3') or T-DNA right border P3 primer (5'-TGGGAAAACCTG GCGTTACCCAACTTAAT-3') and AtCYS6 P4 primer (5'-AGGGACTAGTCATGGTGTTGCTCCG-3') (Fig. 4b).

Promoter-GUS fusion and histochemical analysis

For promoter analysis in transgenic plants, the putative AtCYS6 promoter sequence $(-1,268$ to $+25 \mathrm{bp}$ from the ATG translation start codon) was amplified by PCR from Arabidopsis genomic DNA using EX taq polymerase (Takara, Shiga, Japan) and the forward primer
5'-ACCAATCAAGGAACTTACGAATATTCCAG-3' and the reverse primer 5'-ATTCTCACCTTTATTTGGTT CGGTGCCCTG-3'. PCR amplicons were cloned into the vector pGEM-T Easy (Promega, Madison, WI, USA) and sequenced to confirm the fidelity of amplification. The sequence was then analyzed with the PlantCARE (http:// bioinformatics.psb.ugent.be/webtools/plantcare/html/) and PLACE (http://www.dna.affrc.go.jp/PLACE) databases. The putative promoter sequence was digested from the pGEM-T Easy vector with HindIII/XbaI and subcloned into the same sites of pBI121 (Clontech, Palo Alto, CA, USA). The construct $\left(\mathrm{P}_{\text {AtCYS6: }}: G U S\right)$ was transformed into Agrobacterium tumefaciens strain GV3101 containing the pSOUP vector (Hellens et al. 2000) by electroporation and then into $A$. thaliana Col-0 by the floral dip method (Clough and Bent 1998).

Histochemical staining to detect GUS expression in

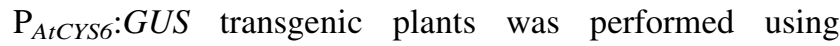
5-bromo-4-chloro-3-indolyl- $\beta$-D-glucuronide (X-gluc; Duchefa, Haarlem, The Netherlands) as a substrate (Jefferson et al. 1987). Tissue was harvested and immediately fixed for $30 \mathrm{~min}$ in ice-cold $90 \%$ acetone (Vanderbeld and Snedden 2007), rinsed with water, and incubated in GUS staining solution (100 mM sodium phosphate buffer [pH 7.0], $0.5 \mathrm{mM}$ EDTA, $0.1 \%$ Triton $\mathrm{X}-100$ and between 0 [high sensitivity] and $1 \mathrm{mM}$ [standard sensitivity] potassium ferrocyanide and potassium ferricyanide). The histochemical reaction was performed in the dark at $37^{\circ} \mathrm{C}$ for $12 \mathrm{~h}$. The plant materials were cleared by washing with several changes of $80 \%$ ethanol.

\section{Western blot analysis}

The AtCYS6 expression pattern was determined by western blot analysis using the anti-BrCYS1 polyclonal antibody (1:10,000 dilution) as previously described (Hong et al. 2007), followed by the addition of peroxidase-conjugated goat anti-rabbit IgG (1:5,000 dilution) according to the manufacturer's guidelines. Hybridization to protein bands was detected using the ECL Western Blotting Analysis System (GE Healthcare, Buckinghamshire, UK).

To confirm AtCYS6 expression during germination or post-germinative growth in response to germination-related phytohormones, dry seeds were placed into $15-\mathrm{cm}$ Petridishes containing filter papers moistened with or without $10 \mu \mathrm{M}$ ABA or $\mathrm{GA}_{4+7}$ (Sigma, St. Louis, MO, USA). Seeds were primed at $4{ }^{\circ} \mathrm{C}$ for 3 days in the dark and incubated for 12 days under normal conditions $(16 \mathrm{~h}$ of $100 \mu \mathrm{Es}^{-1} \mathrm{~m}^{-2}$ light at $\left.22^{\circ} \mathrm{C}\right)$. Samples were collected throughout this period (Kim et al. 2008), and western blot analysis was performed as described above. 
Reverse transcription (RT)-PCR

For quantitative RT-PCR analysis, total RNA was isolated using TRIzol reagent (Invitrogen, Carlsbad, CA, USA), and cDNA was synthesized from $2 \mu \mathrm{g}$ total RNA using SuperScript II RNase H-reverse transcriptase (Invitrogen). Each cDNA sample was diluted $1: 10$, and $1 \mu \mathrm{l}$ of the diluted cDNA was used for PCR amplification with AtCYS6-specific primer sets (forward primer P1 and reverse primer P4, Fig. 4b). PCR amplification of Arabidopsis Actin2 (At3g18780) using gene-specific primers (forward primer: $\quad 5^{\prime}$-TCGGTGGTTCCATTCTTGCT-3'; reverse primer: $\quad 5^{\prime}$-GCTTTTTAAGCCTTTGATCTTGAGAG-3') was performed as a loading control (Lim et al. 2007).

Generation of AtCYS6 over-expressing plants and rescue of the cys6-2 allele

AtCYS6 (At3g12490) cDNA was synthesized using RNA extracted from Arabidopsis flowers. Two micrograms of total RNA was reverse transcribed in a $30 \mu$ reaction volume with $1 \mu \mathrm{g}$ oligo $(\mathrm{dT})_{18}$ primer using MMLV RTase according to the manufacturer's protocol (Toyobo, Osaka, Japan). The gene-specific primers P1 and P4 were used for amplification of AtCYS6 cDNA (Fig. 4b). A PCR product of the predicted size was cloned into pGEM-T Easy and confirmed by DNA sequencing. A $0.74 \mathrm{~kb}$ fragment of AtCYS6 cDNA was inserted in the sense orientation between the cauliflower mosaic virus (CaMV) 35S promoter (35S-P) and the nopaline synthase terminator (noster) in pBI121 (Fig. 4a). Arabidopsis was transformed by the floral dip method, and homozygous $\mathrm{T}_{3}$ lines containing a single T-DNA insertion were used for analyses. To verify the presence of the transgenes and proteins in transformed lines and progeny, RT-PCR was performed using the P1 and $\mathrm{P} 4$ primer set, and protein bands were detected by western blotting as described above.

To complement the cys6-2 allele, a genomic fragment (Fig. 4b) of the AtCYS6 locus encoded by a 3,666 bp genomic PCR amplicon was cloned in the vector pCAMBIA1301 (http://www.cambia.org). This plasmid was transformed into a cys6-2 T-DNA insertional mutant using the floral dip method.

\section{Germination assay and measurement of $\mathrm{CP}$ activity}

To compare germination rates, transgenic and untransformed wild-type Arabidopsis seeds were harvested on the same day. Seeds were surface-sterilized and sown on $0.25 \%(\mathrm{w} / \mathrm{v})$ phyta-gel plates containing half-strength MS medium (1/2MS, $\mathrm{pH} 5.8)$. The plates were placed at $4^{\circ} \mathrm{C}$ for 3 days and then moved to $22^{\circ} \mathrm{C}$ under a long day photoperiod, and germination was scored by microscopy based on radicle emergence. In each experiment, approximately 100 seeds were used, and triplicate experiments were carried out using independent seed lots (Tatematsu et al. 2008; Zheng et al. 2008). CP activity measurements were performed following the methods described in Hong et al. (2007).

\section{Results}

Sequence characterization of the AtCYS6 promoter region

An upstream region including the putative promoter sequence of the AtCYS6 gene was isolated by PCR of genomic DNA, and subsequent sequence analysis with the PLACE (Higo et al. 1999) and PlantCARE (Rombauts et al. 1999) databases revealed several motifs that regulate gene expression and are commonly found in most eukaryotic promoters (Fig. 1). Potential regulatory elements associated with hormone- and stress-related responses that are found in other plant promoters were identified within the AtCYS6 promoter. These include two copies of the ABA-responsive element (ABRE; PyACGTGGC), which is involved in abscisic acid responsiveness (Washio 2003), and pyrimidine boxes (P-box; CTTTT), which are involved in gibberellin responsiveness (Huang et al. 1990). One copy of the MYB binding site (MBS; CAACTG), involved in drought inducibility, together with two MYB recognition elements (MRE; AACCTAA) that mediate response to light (Feldbrügge et al. 1997) were also found in the AtCYS6 promoter sequence. The presence of these motifs indicates that AtCYS6 may be regulated by various cis-acting elements within the promoter as well as by corresponding trans-acting factors (Hong and Hwang 2009).

Spatial and temporal expression of AtCYS6 in Arabidopsis

To precisely define the spatio-temporal expression pattern of AtCYS6, we studied the expression levels of AtCYS6 protein by western blot analysis. As shown in Fig. 2a, AtCYS6 was detected in most plant tissues examined and was particularly prevalent in dry seeds, seedlings and flowers. In 5-day-old seedlings, AtCYS6 was more strongly expressed in root tips than in cotyledons. In mature plants grown in soil, AtCYS6 was barely detectable in rosettes and cauline leaves. These results are in agreement with the expression patterns of several other PhyCys that accumulate more extensively in seeds and seedlings than in fully grown vegetative tissues (Kondo et al. 1990; Abe et al. 1995; Lim et al. 1996; Kuroda et al. 2001; Hong et al. 2007). 
Fig. 1 The nucleotide sequence of the $5^{\prime}$-flanking promoter region and putative cis-acting elements of the AtCYS6 gene. The sequences of the $5^{\prime}$-flanking region and the first exon of AtCYS6 are shown together with a partial amino acid sequence specified by the $5^{\prime}$-end of the AtCYS6 coding region. The numbering of nucleotides relative to the putative transcription initiation site $(+1)$ is shown above. The putative TATA box is identified by $a$ double underline. The deduced amino acid sequences of the first exon are indicated by single initial bold letter codes. ABRE, ABA-responsive element; MBS, MYB binding site; MRE, MYB recognition element; P-box, pyrimidine box

-1268 TAACCAATCAAGGAACTTACGAATATTCCAGTTTCCAAATTATACA -1223

$-12$

$-11$

$-1062$

$-1062$

$-98$

$-90$

$-88$

$-74$

$-662$

$-582$

$-502$

$-422$

$-342$

$-342$

$-262$

$-182$

$-102$

$-22$
CTTTTGAATAATGTCAAGTTTGTGAGTTGTGACTATATATATAGCTATATACTAATTAATTAAGTGGACGGTTACGTCGT $\quad-1143$

GCAGGTGGATGGGCTACAGCACCAGACGGACCATATTCATGGGGCTACTGTTTCAAGCAAGAACAGAATCCTGCTTCAGA -1063

CTACTGTGAACCGAGCGCCACGTGGCCATGCGCATCTGGCAAACGCTACTACGGAAGAGGACCAATGCAACTGTCGTGGA -983

MBS

ACTACAATTACGGTCTATGCGGTAGAGCAATAGGAGTTGACTTACTCAACAACCCTGACCTTGTTGCCAACGACGCAGTG -903

ATCGCTTTCAAAGCCGCGATTTGGTTCTGGATGACTGCTCAGCCTCCCAAACCGTCTTGCCATGCCGTGATAGCCGGCCA -883

GTGGCAGCCTTCAGACGCCGACCGTGCCGCCGGGAGATTACCGGGTTATGGAGTGATTACGAACATCATTAACGGTGGAT -743

TGGAGTGTGGACGTGGCCAAGACGGGAGAGTCGCCGATCGTATAGGGTTTTATCAGAGGTATTGTAACATATTTGGTGTT -663 ABRE

AATCCTGGTGGTAATCTTGATTGTTACAACCAAAGATCCTTCGTTAACGGCCTCCTCGAAGCTGCTATTTAGTAACGAGA $\quad-583$ MRE

GTATTATTATTATAATATAATACGCAGCTTTGTATTATCAAAACAATAATATCAGACTAATAAAACTCTTTCCAATGATG $\quad-503$

TTTCACTGTTTATGTTGCGATTTTATTTCCCATGGGCCTTTTTTAATTGGGCTCTCTAGCAGATGAGAGAAAGACGAAGA -423

P-Box

AAGGTGCATAGATGTTTTAAACTTCGATTACAAGTAAAGGTGTTTGACAATTTCTGTGTGACTCTGCCGACACGTTTCGT $\quad-343$

ATAACGTGCCGCTATCCTCAGCAACGTTTTCATGTGTAATCCAGATTGGTATGAATTTCTATTTAAAATGTTGGTAGCTT $\quad$-263 ABRE

AACCGATTGAGGCCAAATCAAACCAATCTATAACTCGAATCGGTACTCTTTTCTTACAAATGATTCGATTCAATTGGCAT - 183

CCAATCAAATGTAAACCTMTTTTGTTTTAAATCGTAAGAATATTTTCATAAATCAGAGCCTAAACTTAAAACTGTACCAA -103

P-Box

CAAAACCAACATCAAACCAAATGGACACCTTAATCCGGTACACGGCGAATTAACTGTAGAGGATAAAATCAACAATTCGA -23 MRE

TATA-Box

CGAGTAAAACGAAAAATATCTÄAAAATACAGGGCACCGAACCAAATAAAGGTGAGAATGATGAGAAGCCGTTTCTTACTC 58
To further explore this expression pattern, we characterized transgenic plants harboring a transcriptional fusion of the GUS reporter gene and the AtCYS6 promoter $\left(\mathrm{P}_{A t C Y S 6}: G U S\right)$. The 10 transgenic lines that were analyzed revealed similar patterns of GUS expression. These patterns were highly consistent among the transgenic lines, but minor variations in GUS staining intensity were observed among lines with different $\mathrm{P}_{A t C Y S 6}$ :GUS constructs, suggesting mild positional effects of transgene insertion (Vanderbeld and Snedden 2007). $\mathrm{P}_{A t C Y S 6}$ :GUS transgenic Arabidopsis seedlings displayed GUS activity in both cotyledons and root tips (Fig. 2b-c), which decreased dramatically in cotyledons as the seedlings matured and was difficult to detect in rosette and cauline leaves in fully grown plants (Fig. 2d-e), with the exception of root tips. GUS expression in the root tip was prominent in the root apical meristem and root cap (Fig. 2f). These results indicate that AtCYS6 likely plays important roles in seedling growth and that its promoter possesses a complex regulatory mechanism.

After the emergence of the primary inflorescence, relatively weak levels of GUS expression were visible during the early stages of floral development, at stage 9 (Fig. 2g) and stage 12 (Fig. 2h). The growth stages of Arabidopsis have been carefully defined, which allows for an accurate sampling of materials for comparative analysis (Boyes et al. 2001). In open flowers (stage 15), GUS expression was strongly detected in pollen grains in the anthers (Fig. 2i). In weakly expressing transgenic lines, GUS expression in pollen grains was only slightly visible by stage 15 (Fig. 2j), but in more strongly expressing lines was easily visible in pollen grains until dehiscence occurred (Fig. 2k). The increased expression pattern of GUS in pollen grains in anthers during flower development suggests that the AtCYS6 gene might be engaged in microsporogenesis (Charbonnel-Campaa et al. 2000). The observation that $\mathrm{P}_{A t C Y S 6}$ : GUS was particularly abundant in the developing stigmatic papillae of immature siliques (Fig. 2l) suggests that AtCYS6 also modulates the activity of CPs during silique development. The GUS staining patterns in transgenic Arabidopsis were similar to the expression patterns of the AtCYS6 protein (Fig. 2a), and GUS expression analysis revealed more detailed expression patterns of AtCYS6 in plant tissues than was possible to detect by western blotting. All of these results indicate that a 1,268 bp fragment of the AtCYS6 promoter drives expression in seeds, seedlings, meristematic tissues in roots, pollen grains in anthers and stigmatic papillae in 

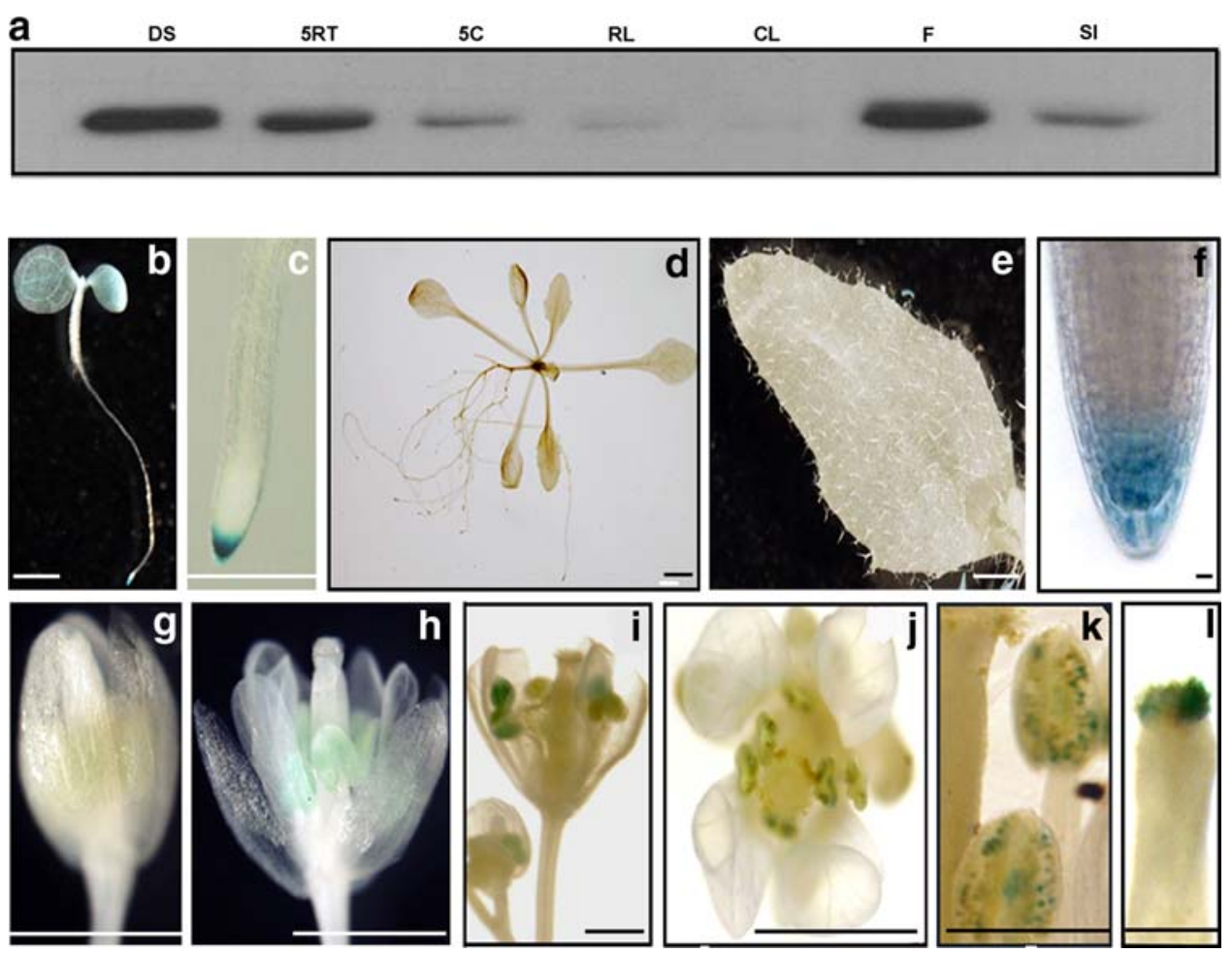

Fig. 2 Western blot analysis and histochemical localization of GUS activity in various organs of transgenic Arabidopsis. a Tissue-specific expression of AtCYS6 in different tissues. Total proteins from dry seeds (DS), root tips from 5-day-old seedlings (5RT), cotyledons from 5-day-old seedlings (5C), rosette leaves (RL), cauline leaves (CL), flowers (F) and siliques (SI) were isolated and subjected to western blot analysis. b Seedling grown on $1 / 2 \mathrm{MSO}$ medium at day 5. c Primary root from a 5-day-old seedling. d Eighteen-day-old transgenic plant grown on soil. e Cauline leaf from a 5-week-old

young siliques. This finding means that the AtCYS6 promoter can modulate the precise transcriptional regulation of specific and developmental expression of AtCYS6 in the seedling, root, flower and siliques of transgenic Arabidopsis. Taken together, these distribution characteristics suggest that AtCYS6 is likely to have a variety of roles and functions in plants in response to complex developmental cues.

\section{Expression of AtCYS6 during seed germination}

The AtCYS6 promoter sequence contains several motifs identical or similar to many well-characterized motifs present in inducible promoters that respond to germinationrelated phytohormones (Fig. 1). The GUS reporter gene driven by the AtCYS6 promoter was expressed primarily in germinating seedlings, especially in organs of embryonic origin, such as cotyledons and primary root tips (Fig. 2). This suggests that regulation of the AtCYS6 promoter may be controlled by germination-related phytohormones, such as $\mathrm{ABA}$ and/or GAs. To test this hypothesis, we examined transgenic plant. f Close-up of a primary root tip with predominant GUS expression in the meristematic tissues. g Floral bud (stage 9). h Young flower (stage 12). i Mature flower (stage 15) in a strongly expressing transgenic line. j Mature flower (stage 15) in a weakly expressing transgenic line. $\mathbf{k}$ Close-up of anthers with predominant GUS expression in pollen grains from a strongly expressing transgenic line. I Young silique. Bars are $1 \mathrm{~mm}$ in (b), (d-e), $500 \mu \mathrm{m}$ in (c), (g-k), $10 \mu \mathrm{m}$ in (f) and $200 \mu \mathrm{m}$ in (l)

the expression levels of AtCYS6 during seed germination and seedling growth, either under standard growth conditions or following treatment with $10 \mu \mathrm{M} \mathrm{ABA}$ or $\mathrm{GA}_{4+7}$, using the anti-BrCYS1 polyclonal antibody (Hong et al. 2007). AtCYS6 expression decreased within 3 days after imbibition on $1 / 2 \mathrm{MS}$ medium without any phytohormones $(1 / 2 \mathrm{MSO})$ or on $1 / 2 \mathrm{MS}$ medium containing $\mathrm{GA}_{4+7}$ $\left(1 / 2 \mathrm{MSGA}_{4+7}\right)$. Conversely, in response to ABA treatment (1/2MSABA), AtCYS6 expression strongly persisted throughout the sampling period and continued to be detected at day 12 (Fig. 3a).

Expression of $\mathrm{P}_{A t C Y S 6}: G U S$ was also examined under the same conditions to evaluate the effect of germinationrelated phytohormones on AtCYS6 expression (Fig. 3b). Like the expression patterns obtained by western blotting, GUS levels essentially disappeared after 7 days in $1 / 2 \mathrm{MSO}$ or $1 / 2 \mathrm{MSGA}_{4+7}$ medium. In contrast, robust GUS expression was detected throughout the sampling period in $1 / 2 \mathrm{MSABA}$ medium. Interestingly, in response to treatment with ABA, GUS was strongly expressed in the apical hook, cotyledon and root tip of seedlings. Taken together, 
Fig. 3 AtCYS6 is expressed in seeds prior to germination and seedling growth. Western blot analysis (a) and $\mathrm{P}_{A t C Y S 6}: G U S$ expression (b) in response to phytohormones during germination and postgermination growth of Arabidopsis. Total proteins were prepared from control (1/2MSO), $10 \mu \mathrm{M}$ ABA $(1 / 2 \mathrm{MSABA})$ or $\mathrm{GA}_{4+7}$ $\left(1 / 2 \mathrm{MSGA}_{4+7}\right)$-treated seeds at the indicated day after imbibition (DAI). Bars are $1 \mathrm{~mm}$ in (b)

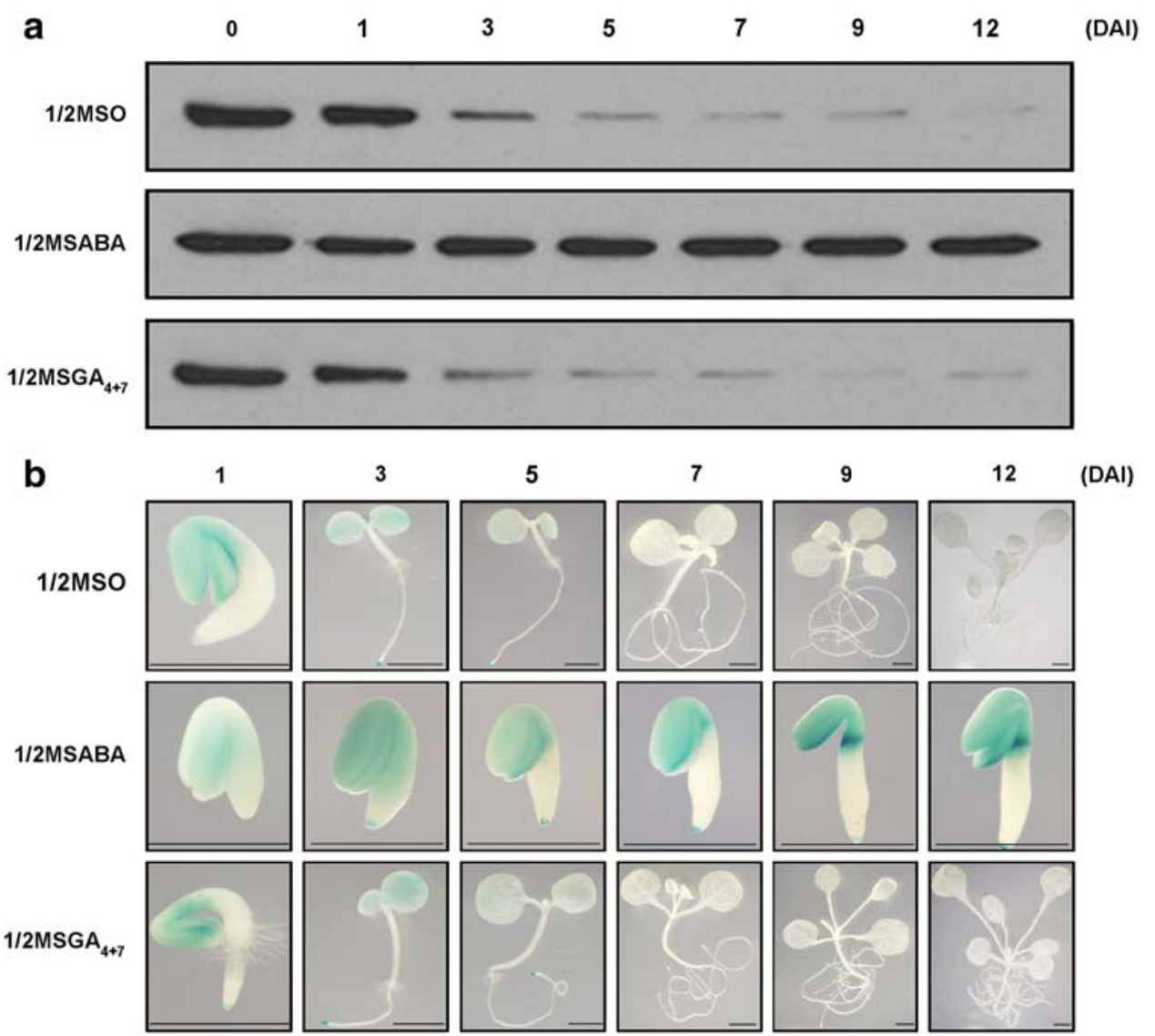

these findings reveal that AtCYS6 expression is continuously maintained by ABA treatment during germination and seedling growth, suggesting that AtCYS6 may play an inhibitory role in these processes.

\section{Over-expression and knock-out mutants in Arabidopsis}

To define the in vivo function of AtCYS6 in seed germination and seedling growth, we transformed Arabidopsis seedlings with a vector carrying a fusion of the CaMV35S promoter and AtCYS6 cDNA ( $\mathrm{P}_{355}$ :AtCYS6; Fig. 4a). From $\mathrm{T}_{1}$ plants, we selected 15 independent lines using selection medium containing kanamycin. PCR analysis revealed the presence of both AtCYS6 and nptII in each plant selected (data not shown). To determine the number of AtCYS6 copies in the transgenic plants, $\mathrm{T}_{1}$ plants were self-pollinated and the progeny $\left(T_{2}\right)$ were allowed to segregate on selection media. Following self-pollination of the $\mathrm{T}_{2}$ lines, three $\mathrm{T}_{3}$ homozygous lines (OX1-OX3) that contained a single T-DNA insertion were selected (Fig. 4c). The presence of AtCYS6 genomic DNA (1.44 kb) and cDNA sequences $(0.74 \mathrm{~kb})$ was verified by genomic DNA PCR using gene-specific primers. In addition, the in vivo function of AtCYS6 was analyzed using a T-DNA insertional mutant that disrupts the AtCYS6 locus. A T-DNA insertion of AtCYS6 was identified in the Salk collection (Columbia background; donor stock number SALK_027847) and was designated cys6-2 (Fig. 4b). The DNA sequence of the T-DNA flanking region of cys6-2 indicated that the insertion was in the third intron of AtCYS6. To confirm these data, genomic fragments adjacent to the left border of the T-DNA insertion were sequenced. Sequence analysis confirmed that the T-DNA insertion was located at nucleotide 969 of the AtCYS6 gene (numbering begins at the ATG start codon; Fig. 4b). To identify plants homozygous for the T-DNA insertion, genomic DNA was obtained from kanamycin-resistant cys6-2 seedlings and subjected to PCR analysis (Fig. 4d). Genomic PCR analysis detected a $1.44 \mathrm{~kb}$ AtCYS6 fragment in wild-type (WT) plants; however, PCR analysis using the T-DNA left border primer (P2) or right border primer (P3), did not detect an amplicon. For the cys6-2 mutant allele, PCR amplicons that encompass the T-DNA insertion site were not detected; however, when the P1 primer and the T-DNA left border primer $(\mathrm{P} 2)$, or the $\mathrm{P} 4$ primer and right border primer $(\mathrm{P} 3)$, were used, amplicons were evident.

Fourteen-day-old untransformed WT plants, the overexpression lines (OX1-OX3), the cys6-2 mutant line and a rescued cys6-2 line (CM) were selected for examination of AtCYS6 transcripts levels by RT-PCR (Fig. 4e). AtCYS6 

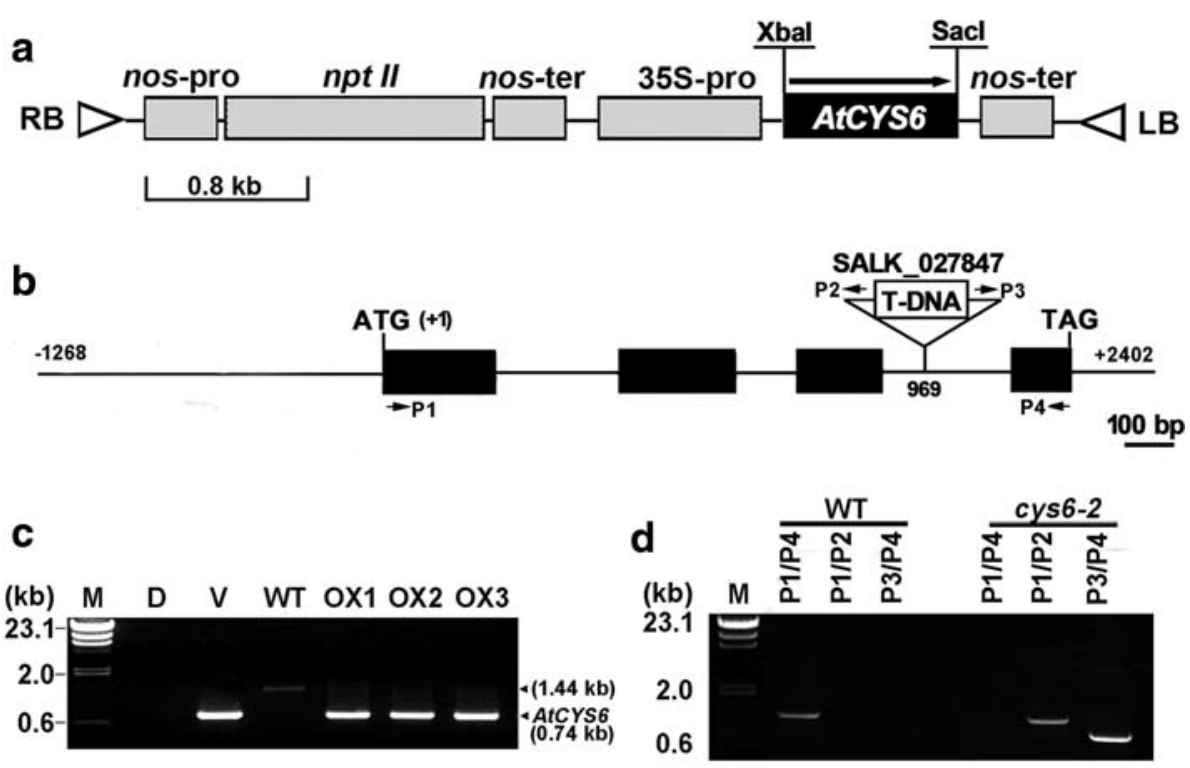

e

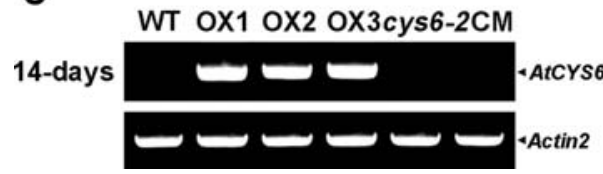

f

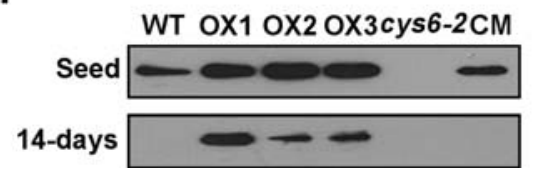

Fig. 4 The expression of AtCYS6 in transgenic plants. a Structure of the $\mathrm{P}_{35 s}$ :AtCYS6 construct for AtCYS6 cDNA expression. The gene encoding bacterial neomycin phosphotransferase II (npt II), which is regulated by the nopaline synthase gene promoter (nos-pro) and $3^{\prime}$ terminator (nos-ter), serves as a selectable marker for Arabidopsis transformation. AtCYS6 is regulated by the CaMV $35 \mathrm{~S}$ promoter (35Spro). LB and RB indicate left and right T-DNA borders, respectively. b Schematic diagram of the AtCYS6 gene and localization of the T-DNA insertion in the cys6-2 allele (SALK_027847). Numbering begins at the ATG translation start codon $(+1)$. Exons are represented by black boxes. The position of the T-DNA insertion is indicated in a bracket with respect to the ATG start codon. Numbered arrows show the positions and orientations of the P1 through $\mathrm{P} 4$ primers that were used for genotyping and PCR expression analysis. $\mathbf{c}$ PCR analysis of

cDNA was strongly detectable only in OX1-OX3 plants. Protein expression in seeds and in 14-day-old progeny of homozygous transgenic lines was also analyzed by immunoblotting (Fig. 4f). A single $22.4 \mathrm{kDa}$ band corresponding to AtCYS6 was detected in WT, OX1-OX3 and CM seeds, but not in cys6-2 seeds. Both AtCYS6 transcripts and protein were undetectable in 14-day-old plants (Fig. 4e-f), which is in agreement with the finding that AtCYS6 accumulates more in seeds and seedlings than in mature plants. RT-PCR and western blot analysis revealed that the cys6-2 mutation resulted in the complete loss of AtCYS6 expression at the transcription (Fig. 4e) and translation levels (Fig. 4f), respectively. The $5 \mathrm{~kb}$ T-DNA insertion potentially disrupts splicing or affects the stability of the AtCYS6 transcript. Therefore, the cys6-2 mutant likely contains a null allele of AtCYS6. untransformed wild-type and transgenic Arabidopsis plants. The presence of AtCYS6 was verified by genomic PCR using the P1 and P4 primers. $M$ molecular mass markers; $D$ double-distilled water as template; $V \mathrm{P}_{35 s}$ :AtCYS6 vector as template; $W T$ untransformed wildtype plants; OX1-OX3, $\mathrm{P}_{35 s}$ :AtCYS6 lines no. 1-3. d Diagnostic PCR of the T-DNA inserted in the AtCYS6 locus. Genomic DNA from WT plants and cys6-2 homozygotes was used. PCR primers are indicated above each lane. e RT-PCR analysis of AtCYS6 transcripts in 14-dayold WT, OX1-OX3, cys6-2 and KO transgenic plants rescued with the AtCYS6 gene (CM). The P1 and P4 primer set used for RT-PCR is shown in (b). The Actin2 gene (At3g18780) served as a control. f Western blot analysis of AtCYS6 in seeds (upper panel) and 14-dayold progeny of transgenic plants (lower panel)

Over-expression of AtCYS6 retards seed germination and seedling growth

We germinated seeds from OX2, cys6-2 and CM transgenic lines as well as from WT plants on $1 / 2 \mathrm{MSO}$ medium. As shown in Fig. 5a, OX2 seeds germinated at a slower rate than WT and CM seeds, whereas cys6-2 seeds germinated slightly faster. This raises the possibility that AtCYS6 may inhibit stored CP activity in seeds and during seedling growth, since transgenic plants that over-express AtCYS6 displayed retarded seed germination. Therefore, we measured and quantified endogenous CP activity in transgenic seeds and during seed germination (Fig. 5b). In OX2 seeds, endogenous CP activity was lower than in WT or cys6-2 seeds. Following germination, endogenous CP enzymatic activity increased slightly in OX2 seedlings at $8 \mathrm{~h}$; 

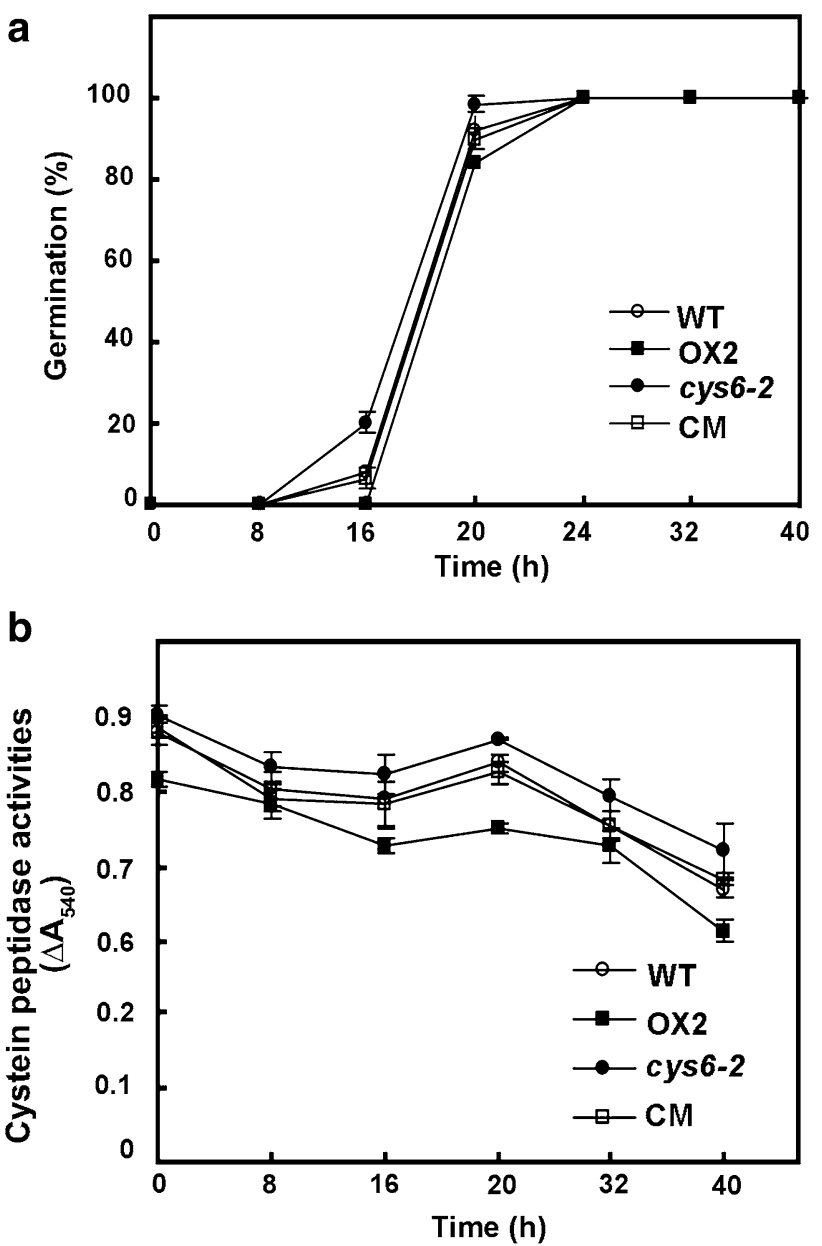

Fig. 5 Comparison of germination and post-germination growth in transgenic AtCYS6 plants. a Time course of germination for freshly harvested seeds of untransformed wild-type (WT), over-expression (OX2), and knock-out (cys6-2) plants, and rescued lines (CM). b Analysis of endogenous $\mathrm{CP}$ activity during seed germination and post-germination growth. Endogenous $\mathrm{CP}$ activity was measured in protein extracted from seeds and seedlings at different stages. Seeds used in each experiment originated from the same seed batch. Data are means \pm S.E. from at least three independent experiments

however, this enzymatic activity was lower than that of WT and cys6-2 seedlings. The lower CP enzymatic activity might be a result of the delayed germination of OX2 seeds due to the accumulation of AtCYS6. This suggests that over-expression of AtCYS6 caused the decrease in stored $\mathrm{CP}$ activity during germination.

As AtCYS6 over-expressing transgenic seedlings grew slowly, we sought to explore the function of AtCYS6 in post-germination growth. We compared seedling growth rates in transgenic plants and found that the primary root elongation pattern of OX2 plants was delayed compared to that of WT and CM plants, whereas cys6-2 roots grew slightly faster (Fig. 6a-b). The fresh weights of transgenic Arabidopsis seedlings paralleled root length patterns (Fig. 6c). These data indicate that the over-expression of
AtCYS6 also retards seedling growth. This evidence strongly implicates a key role for AtCYS6 both in the regulation of stored CPs in vivo and in the control of seedling growth.

\section{Discussion}

Cysteine proteinase and PhyCys interactions have been repeatedly implicated in the control of germination and seedling growth (Shutov and Vaintraub 1987; Müntz 1996; Okamoto and Minamikawa 1999; Kato et al. 2003; Zakharov et al. 2004). In light of this, we considered the possibility that AtCYS6 might be regulated by germinationrelated phytohormones and play crucial roles in the regulation of germination and seedling growth. As an initial step toward understanding the regulatory mechanisms that control AtCYS6 gene expression, we analyzed the upstream promoter sequences of the AtCYS6 gene (Fig. 1). This analysis revealed that AtCYS6 contains several interesting putative cis-elements. Among them, we observed important cis-acting elements that are responsive to germinationrelated phytohormones and may direct seed germination and seedling growth, including ABREs and P-boxes. The ABRE motif and the P-box motif have been identified as binding sites for ABRE binding proteins (AREBs) (LopezMolina and Chua 2000) and Dof (DNA with one finger) transcription factors (Yanagisawa 2002), respectively. The distinct cis-elements identified in the AtCYS6 promoter sequences prompted us to analyze the $5^{\prime}$-upstream regions of AtCYS6 using transgenic Arabidopsis plants carrying a GUS fusion to the putative promoter region. Analysis of transgenic Arabidopsis carrying the AtCYS6 promoterGUS construct revealed that AtCYS6 is expressed predominantly in seedling cotyledons and root meristematic tissues (Fig. 2). Additionally, the levels of AtCYS6 and GUS were rapidly decreased by treatment with $\mathrm{GA}_{4+7}$ or water, whereas expression strongly persisted following ABA treatment (Fig. 3). These results concur with the findings of Martínez et al. (2003) who showed that ABA represses the steady-state levels of the cathepsin B-like CP gene (CatB) and induces PhyCys-encoding gene (Icy) expression. Thus, it is tempting to speculate that the two putative ABREs in the AtCYS6 promoter (Fig. 1) may be responsible for controlling AtCYS6 expression by ABA (Fig. 3). It is possible that AtCYS6 is regulated by transcriptional regulators that are activated in response to $\mathrm{ABA}$ signaling. A more detailed functional analysis is necessary to determine which ABRE is responsible for $\mathrm{ABA}$ regulation of the AtCYS6 promoter.

We further investigated the inhibitory activities of AtCYS6 during germination and seedling growth using AtCYS6 over-expressing ( $\mathrm{P}_{355}:$ AtCYS6) and knock-out 
Fig. 6 Comparison of seedling growth in transgenic AtCYS6 plants. a Phenotypes are shown for 5-day-old untransformed wild-type (WT), overexpression (OX2), and knockout (cys6-2) plants, and rescued lines (CM). Bar is $1 \mathrm{~cm}$. b Quantitative analysis of primary root lengths of 5-day-old seedlings from each transgenic line. c Fresh weights of 5-day-old WT and transgenic plant seedlings. The average fresh weight was estimated in three independent experiments using three to five of the 20 seedlings used in each experiment. Data are presented as the means $\pm \mathrm{SE}$. from at least three independent experiments a
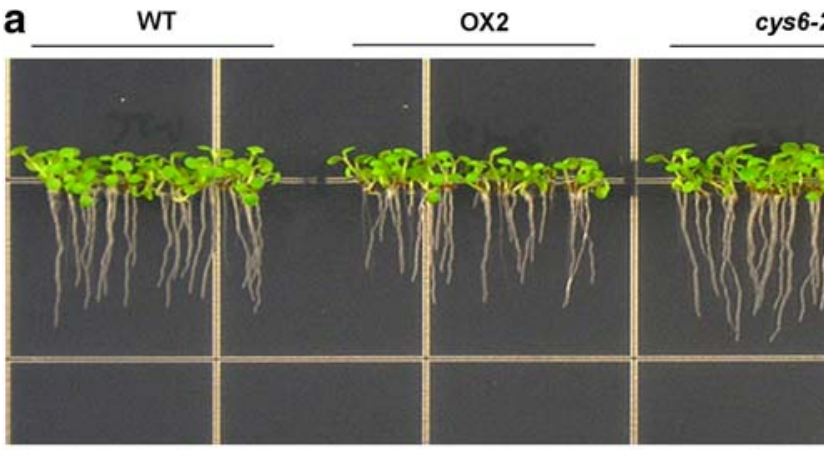

cys6-2 CM

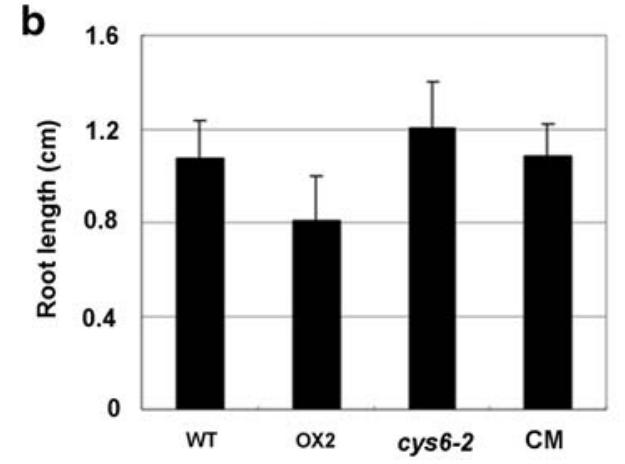

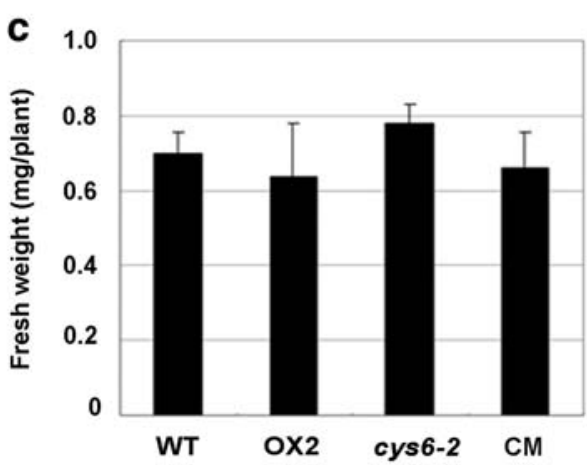

(cys6-2) transgenic plants (Fig. 4). Transgenic Arabidopsis lines $\left(\mathrm{T}_{3}\right)$ that constitutively express AtCYS6 displayed weak growth inhibition in seedlings, whereas seedling growth was enhanced by suppression of AtCYS6 (Figs. 5, 6). Additionally, CP activity stored in seeds was inhibited by AtCYS6 in $\mathrm{P}_{355}$ :AtCYS6 transgenic plants. These findings reconfirmed that persistent AtCYS6 expression modulates the activity of papain-like CPs in transgenic Arabidopsis seeds and seedlings. Several PhyCys with a molecular mass of $\approx 23 \mathrm{kDa}$ have a C-terminal extension that has been shown to be involved in the inhibition of legumains (Zakharov et al. 2004). We observed that AtCYS6 (22.4 kDa) has an extended C-terminus and modulates the activity of commercial papaya latex papain. This suggests that AtCYS6 might inhibit not only the activity of papain-like CPs but also that of legumains, which are generally considered to be the major endopeptidases responsible for the degradation of seed storage proteins during early seedling growth (Zakharov et al. 2004).

The data from our study indicate that AtCYS6 plays a critical role not only in seed germination but also in seedling growth. It is possible that AtCYS6 also suppresses the action of endogenous papain-like CPs during germination and seedling growth. To confirm this, it will be necessary to define the interactions of various endogenous papain-like CPs with PhyCys in Arabidopsis. Further investigation of Arabidopsis PhyCy isoforms should improve our understanding of the biological roles and functions of PhyCys in seed germination and seedling growth.
Acknowledgments This work was supported by the Environmental Biotechnology National Core Research Center Program (R15-2003012-01001-0). J.E. Hwang and J.H. Je were supported by a BK21 fellowship from the MEST, Republic of Korea.

Open Access This article is distributed under the terms of the Creative Commons Attribution Noncommercial License which permits any noncommercial use, distribution, and reproduction in any medium, provided the original author(s) and source are credited.

\section{References}

Abe M, Abe K, Domoto C, Arai S (1995) Two distinct species of corn cystatin in corn kernels. Biosci Biotech Biochem 59:756-758

Boyes DC, Zayed AM, Ascenzi R, McCaskill AJ, Hoffman NE, Davis KR, Görlach J (2001) Growth stage-based phenotypic analysis of Arabidopsis: a model for high throughput functional genomics in plants. Plant Cell 13:1499-1510

Charbonnel-Campaa L, Lauga B, Combes D (2000) Isolation of a type 2 metallothionein-like gene preferentially expressed in the tapetum in Zea mays. Gene 254:199-208

Clough SJ, Bent AF (1998) Floral dip: a simplified method for Agrobacterium-mediated transformation of Arabidopsis thaliana. Plant J 16:735-743

Corre-Menguy F, Cejudo FJ, Mazubert C, Vidal J, Lelandais-Brire C, Torres G, Rode A, Hartmann C (2002) Characterization of the expression of a wheat cystatin gene during caryopsis development. Plant Mol Biol 50:687-698

Feldbrügge M, Sprenger M, Hahlbrock K, Weisshaar B (1997) $P c$ MYB1, a novel plant protein containing a DNA-binding domain with one MYB repeat, interacts in vivo with a lightregulatory promoter unit. Plant J 11:1079-1093

Gaddour K, Vicente-Carbajosa J, Lara P, Isabel-Lamoneda I, Díaz I, Carbonero P (2001) A constitutive cystatin-encoding gene from 
barley (lcy) responds differentially to abiotic stimuli. Plant Mol Biol 45:599-608

Hellens RP, Edwards EA, Leyland NR, Bean S, Mullineaux PM (2000) pGreen: a versatile and flexible binary Ti vector for agrobacterium-mediated plant transformation. Plant Mol Biol 42:819-832

Higo K, Ugawa Y, Iwamoto M, Korenaga T (1999) Plant cis-acting regulatory DNA elements (PLACE) database. Nucleic Acids Res 27:297-300

Hong JK, Hwang BK (2009) The promoter of the pepper pathogeninduced membrane protein gene CaPIMP1 mediates environmental stress responses in plants. Planta 229:249-259

Hong JK, Hwang JE, Lim CJ, Yang KA, Jin ZL, Kim CY, Koo JC, Chung WS, Lee KO, Lee SY, Cho MJ, Lim CO (2007) Overexpression of Chinese cabbage phytocystatin 1 retards seed germination in Arabidopsis. Plant Sci 172:556-563

Huang N, Sutliff TD, Litts JC, Rodriguez RL (1990) Classification and characterization of the rice $\alpha$-amylase multigene family. Plant Mol Biol 14:655-668

Jefferson RA, Kavanagh TA, Bevan MW (1987) GUS fusions: betaglucuronidase as a sensitive and versatile gene fusion marker in higher plants. EMBO J 6:3901-3907

Kato H, Sutoh K, Minamikawa T (2003) Identification, cDNA cloning and possible roles of seed-specific rice asparaginyl endopeptidase, REP-2. Planta 217:676-685

Kim ST, Kang SY, Wang Y, Kim SG, hwang DH, Kang KY (2008) Analysis of embryonic proteome modulation by GA and ABA from germinating rice seeds. Proteomics 8:3577-3587

Kondo H, Abe K, Nishimura I, Watanabe H, Emori Y, Arai S (1990) Two distinct cystatin species in rice seeds with different specificities against cysteine proteinases. J Biol Chem 265:15832-15837

Koornneef M, Bentsink L, Hilhorst H (2002) Seed dormancy and germination. Cur Opin Plant Biol 5:33-36

Kuroda M, Kiyosaki T, Matsumoto I, Misaka T, Arai S, Abe K (2001) Molecular cloning, characterization, and expression of wheat cystatins. Biosci Biotech Biochem 65:22-28

Lim CO, Lee SI, Chung WS, Park SH, Hwang I, Cho MJ (1996) Characterization of a cDNA encoding cysteine proteinase inhibitor from Chinese cabbage (Brassica campestris L. ssp. pekinensis) flower buds. Plant Mol Biol 30:373-379

Lim CJ, Hwang JE, Chen H, Hong JK, Yang KA, Choi MS, Lee KO, Chung WS, Lee SY, Lim CO (2007) Over-expression of the Arabidopsis DRE/CRT-binding transcription factor DREB2C enhances thermotolerance. Biochem Biophys Res Commun 362:431-436

Lopez-Molina L, Chua NH (2000) A null mutation in a bZIP factor confers ABA-insensitivity in Arabidopsis thaliana. Plant Cell Physiol 41:541-547
Martínez M, Díaz I (2008) The origin and evolution of plant cystatins and their target cysteine proteinases indicate a complex functional relationship. BMC Evol Biol 8:198-209

Martínez M, Rubio-Somoza I, Carbonero P, Díaz I (2003) A cathepsin B-like cysteine protease gene from Hordeum vulgare (gene $C a t B$ ) induced by GA in aleurone cells is under circadian control in leaves. J Exp Bot 54:951-959

Martínez DE, Bartoli CG, Grbic V, Guiamet JJ (2007) Vacuolar cysteine proteases of wheat (Triticum aestivum L.) are common to leaf senescence induced by different factors. J Exp Bot 58:1099-1107

Müntz K (1996) Proteases and proteolytic cleavage of storage proteins in developing and germinating dicotyledonous seeds. $\mathbf{J}$ Exp Bot 47:605-622

Murashige T, Skoog F (1962) A revised medium for rapid growth and bioassays with tobacco tissue culture. Physiol Plant 15:473-497

Okamoto T, Minamikawa T (1999) Molecular cloning and characterization of Vigna mungo processing enzyme I (VmPE-1), an asparaginyl endopeptidase possibly involved in post-translational processing of a vacuolar cysteine endopeptidase (SH-EP). Plant Mol Biol 39:63-73

Rombauts S, Déhais P, Montagu MV, Rouzé P (1999) PlantCARE, a plant cis-acting regulatory element database. Nucleic Acids Res 27:295-296

Shutov AD, Vaintraub IA (1987) Degradation of storage proteins in germinating seeds. Phytochemistry 26:1557-1566

Tatematsu K, Nakabayashi K, Kamiya Y, Nambara E (2008) Transcription factor AtTCP14 regulates embryonic growth potential during seed germination in Arabidopsis thaliana. Plant J 53:42-52

Vanderbeld B, Snedden WA (2007) Developmental and stimulusinduced expression patterns of Arabidopsis calmodulin-like genes CML37, CML38 and CML39. Plant Mol Biol 64:683-697

Washio K (2003) Functional dissections between GAMYB and Dof transcription factors suggest a role for protein-protein associations in the gibberellins-mediated expression of the RAmylA gene in the rice aleurone. Plant Physiol 133:850-863

Yanagisawa S (2002) The Dof family of plant transcription factors. Trends Plant Sci 7:555-560

Zakharov A, Carchilan M, Stepurina T, Rotari V, Wilson K, Vaintraub I (2004) A comparative study of the role of the major proteinases of germinated common bean (Phaseolus vulgaris L.) and soybean (Glycine $\max$ (L.) Merrill) seeds in the degradation of their storage proteins. J Exp Bot 55:2241-2249

Zheng BS, Rönnberg E, Viitanen L, Salminen TA, Lundgren K, Morita T, Edqvist J (2008) Arabidopsis sterol carrier protein-2 is required for normal development of seeds and seedlings. J Exp Bot 59:3485-3499 